\title{
RESTRICTED MEAN VALUES AND HARMONIC FUNCTIONS
}

BY

\author{
JOHN R. BAXTER
}

\begin{abstract}
A function $h$ defined on a region $R$ in $\mathbf{R}^{n}$ will be said to possess a restricted mean value property if the value of the function at each point is equal to the mean value of the function over one open ball in $R$, with centre at that point. It is proved here that this restricted mean value property implies $h$ is harmonic under certain conditions.
\end{abstract}

1. Introduction. Let $R$ be a region in $\mathbf{R}^{n}$. For each $x \in R$, let $S(x)$ be a ball in $R$ with centre $x$. Suppose $h$ is a function defined on $R$ such that

$$
h(x)=[\mu(S(x))]^{-1} \int_{S(x)} h(y) \mu(d y)
$$

for each $x$ in $R, \mu$ being the ordinary Lebesgue measure on $\mathbf{R}^{n}$. In this case $h$ will be said to possess the restricted mean value property with respect to the balls $S(x)$. The purpose of this paper is to show that, under certain conditions, (1.1) implies that $h$ is harmonic. It was proved in the paper of Akcoglu and Sharpe [1] that if $h$ is bounded, if $R$ is the unit disc in $\mathbf{R}^{2}$, and if for each $x \in R, S(x)$ is the largest disc in $R$ with centre $x$, then (1.1) implies $h$ is harmonic. The results in this paper give the following theorem.

THEOREM (1.2). Suppose (a) $h$ is bounded and measurable;

(b) $\boldsymbol{R}$, the closure of $R$ in $\mathbf{R}^{n}$, is a compact $C^{1}$ manifold with boundary;

(c) the radius of $S(x)$ is a measurable function of $x$ and is greater than some fixed fraction of the distance from $x$ to $R^{c}$, for all $x$ in $R$.

Then (1.1) implies $h$ is harmonic on $R$.

2. Restatement of the problem. Let $F(x, y)$ be a measurable function defined on

\section{$R \times R$. Suppose}

(2.1) $F(x, y)$ is bounded in $x$ for each fixed $y$, and

(2.2) $\int|F(x, y)| \mu(d y) \leqq 1$ for each $x$.

Define a linear operator $T$ on $\mathscr{L}_{1}(R, \mu)$ by the equation

(2.3) $T f(y)=\int f(x) F(x, y) \mu(d x)$ for each $y \in R$ and $f \in \mathscr{L}_{1}(R, \mu)$.

Received by the editors January 18, 1971.

AMS 1970 subject classifications. Primary 31B05; Secondary $60 \mathrm{~J} 45$.

Key words and phrases. Harmonic, mean value, positive $\mathscr{L}_{1}$-operator, dissipative, limit at boundary.

Copyright (C) 1972, American Mathematical Society 
It is easy to show that $T$ is well defined and $\|T\| \leqq 1$. Clearly

(2.4) $T^{*} h(x)=\int h(y) F(x, y) \mu(d y)$ for each $x \in R$ and $h \in \mathscr{L}_{\infty}(R, \mu)$.

In particular, suppose

(2.5) $F(x, y)=[\mu(S(x))]^{-1} \chi_{S(x)}(y)$, where (1.2(c)) holds.

Then (2.1) and (2.2) are satisfied. (2.4) becomes

(2.6) $T^{*} h(x)=[\mu(S(x))]^{-1} \int_{S(x)} h(y) \mu(d y)$.

Thus (1.1) is equivalent to

(2.7) $T^{*} h=h$.

To prove Theorem (1.2) is therefore the same as showing that any function left unaltered by $T^{*}$ is harmonic. A proof can be given for a more general $F(x, y)$ than that defined by (2.5). We will now list the properties which will be required of $F(x, y)$ in addition to (2.1) and (2.2). We require

(2.8) $T^{*} h=h$ for all $h$ which are harmonic on $R$.

We require that $\exists \alpha>0, c_{1}>0$ such that, $\forall x \in R$,

(2.9) $F(x, y) \geqq \alpha\left[\mu\left(S_{c_{1} d\left(x, R^{c}\right)}(x)\right)\right]^{-1}$ on $S_{c_{1} d\left(x, R^{c}\right)}(x)$.

Here $S_{r}(p)$ denotes the ball of radius $r$ and centre $p$, and $d\left(x, R^{c}\right)$ denotes the distance from $x$ to $R^{c}$. In future we will write $\mu\left(S_{c}(p)\right)$ as $m(c)$, for brevity.

Let $B^{r}=\left\{x \mid x \in R, d\left(x, R^{c}\right)<r\right\}$ for each $r>0$.

The next property that $F(x, y)$ must have is best expressed in terms of $T$. It might be called the "boundary-strip" property. Intuitively, it means that if one chooses a sufficiently broad strip between a function $f$ and the boundary of $R$, then the operator $T$ cannot make the function jump over the strip. In precise terms:

For every $\varepsilon>0$, a number $\lambda>0$ can be found such that, for any number $d>0$ and any $f \geqq 0$ in $\mathscr{L}_{1}(R, \mu)$,

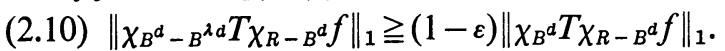

It is easy to see that if $(2.5)$ holds, then $(2.10)$ is true. Using radial coordinates, one finds it is sufficient to have $(1-\lambda)^{n}(2 \lambda)^{-n} \geqq 1-\varepsilon$.

THEOREM (2.11). If (1.2(b)), (2.1), (2.2), (2.8), (2.9), and (2.10) are true, then any function left unaltered by $T^{*}$ is harmonic.

The proof of Theorem (2.11) is completed in $\S 5$, where the main lemma, Lemma (5.4), is given. Some simpler lemmas make up the other sections. To conclude this section, some properties of $T$ are proved, which depend only upon (2.1), (2.2), and (2.8).

We know that $\|T\| \leqq 1$ and $T^{*} h=h$ for every bounded harmonic function. Then, in particular,

(2.12) $\int f d \mu=\int T f d \mu$ for every $f$ in $\mathscr{L}_{1}(R, \mu)$.

Hence

(2.13) $\|T\|=1$, and for every $f \geqq 0$ in $\mathscr{L}_{1}(R, \mu)$,

(2.14) $T f \geqq 0$ and $\|f\|_{1}=\|T f\|_{1}$.

Each function $f \geqq 0$ in $\mathscr{L}_{1}(R, \mu)$ defines an absolutely continuous measure and 
may be pictured as having a certain mass. When $T$ acts on $f$, it pushes its mass about but does not destroy any of its mass.

Let $E$ be a measurable subset of $R$. Define $T_{E}$ on $\mathscr{L}_{1}(R, \mu)$ by

(2.15) $T_{E} f=\chi_{E} f+T_{\chi_{E}} c f$ for each $f$ in $\mathscr{L}_{1}(R, \mu)$.

One may regard $T_{E}$ intuitively as that partial operation of $T$ which holds fixed whatever mass of a function lies on $E$, and moves the remaining mass of the function as much as possible.

Clearly,

(2.16) $\chi_{E} T_{E}^{k} f$ increases to a limit, $\Gamma_{E} f$, for each $f \geqq 0$ in $\mathscr{L}_{1}(R, \mu)$, as $k \rightarrow \infty$.

$\Gamma_{E}$ may be extended to a linear operator on $\mathscr{L}_{1}(R, \mu)$. Then

(2.17) $\left\|\Gamma_{E}\right\| \leqq 1$ and $\Gamma_{E} f=0$ on $E^{c}$ for any $f$.

If $f \geqq 0$, then $\Gamma_{E} f \geqq 0$.

Intuitively, $\Gamma_{E} f$ is the end result of pushing more and more of the mass of $f$ onto $E$, to the greatest degree possible, and then disregarding that part of $f$ which will not leave $E^{c}$.

Lemma (2.18). Let $E$ and $F$ be measurable subsets of $R$, with $E \subset F$. Then $\Gamma_{E}$ $=\Gamma_{E} \Gamma_{F}$.

Proof. It is sufficient to show $\Gamma_{E} f=\Gamma_{E} \Gamma_{F} f$ for $f \geqq 0$ in $\mathscr{L}_{1}(R, \mu)$.

It will be shown by induction that

(2.19) $\chi_{E} T_{E}^{k} \chi_{F} T_{F}^{l} f \geqq \chi_{E} T_{E}^{k} f$ whenever $l \geqq k$.

For $k=0,(2.19)$ reduces to $\chi_{E} T_{F}^{l} f \geqq \chi_{E} f$, which is clearly true.

Assume (2.19) is true for some $k \geqq 0$. Then, for $l \geqq k+1$,

$$
\begin{aligned}
\chi_{E} T_{E}^{k+1} \chi_{F} T_{F}^{l} f & =\chi_{E} T_{E}^{k+1} \chi_{F} T_{F}^{l} \chi_{F} f+\chi_{E} T_{E}^{k+1} \chi_{F} T_{F}^{l} \chi_{F} c f \\
& =\chi_{E} T_{E}^{k+1} \chi_{F} f+\chi_{E} T_{E}^{k+1} \chi_{F} T_{F}^{l-1} T_{E} \chi_{F} c f \\
& \geqq \chi_{E} T_{E}^{k+1} \chi_{F} f+\chi_{E} T_{E}^{k} \chi_{F} T_{F}^{l-1} T_{E} \chi_{F} c f \\
& \geqq \chi_{E} T_{E}^{k+1} \chi_{F} f+\chi_{E} T_{E}^{k} T_{E} \chi_{F} c f \quad \text { (using the induction hypothesis) } \\
& =\chi_{E} T_{E}^{k+1} f .
\end{aligned}
$$

Thus (2.19) holds with $k$ replaced by $k+1$, so (2.19) is true for all $k$.

Letting $l \rightarrow \infty$ and then $k \rightarrow \infty$ in (2.19),

(2.20) $\Gamma_{E} \Gamma_{F} f \geqq \Gamma_{E} f$.

On the other hand,

(2.21) $\chi_{E} T_{E}^{k+l} f \geqq \chi_{E} T_{E}^{k} \chi_{F} T_{F}^{l} f$ for all $k \geqq 0, l \geqq 0$.

In fact

(2.22) $\chi_{E} T_{E}^{k+l} f \geqq \chi_{E} T_{E}^{k} T_{F}^{l} f$ for all $k \geqq 0, l \geqq 0$.

(2.22) can be proved by induction on $l$.

Letting $k \rightarrow \infty$ and then $l \rightarrow \infty$ in (2.21),

(2.23) $\Gamma_{E} f \geqq \Gamma_{E} \Gamma_{F} f$.

Thus the lemma is proved.

The definitions of $T$ and of the partial applications of $T$ follow the paper of Akcoglu and Sharpe [1]. 
It is convenient to express (2.10) again in terms of $\Gamma$ rather than $T$.

Lemma (2.24). For every $\varepsilon>0$, a number $\lambda>0$ can be found such that, for any number $d>0$ and any $f \geqq 0$ in $\mathscr{L}_{1}(R, \mu)$,

$$
\left\|\chi_{B^{d}-B^{2 d}} \Gamma_{B^{d}} \chi_{R-B^{d}} f\right\|_{1} \geqq(1-\varepsilon)\left\|\Gamma_{B^{d}} \chi_{R-B^{d}} f\right\|_{1} .
$$

Proof. Choose $\lambda$ as given by (2.10). By induction,

$$
\left\|\chi_{B^{d}-B^{d d}} T_{B^{d}}^{k} \chi_{R-B^{d}} f\right\|_{1} \geqq(1-\varepsilon)\left\|\chi_{B^{d}} T_{B^{d}}^{k} \chi_{R-B^{d}} f\right\|,
$$

for all $k \geqq 1$. Letting $k \rightarrow \infty$, the result follows.

\section{Dissipation and cancellation properties.}

Lemma (3.1). Let $E$ be a measurable subset of $R$ with $d\left(E, R^{c}\right)=d>0$. Then, for each $f \geqq 0$ in $\mathscr{L}_{1}(R, \mu)$ such that $f=0$ outside $E$,

$$
\int f(y)|y|^{2} \mu(d y)+\left(\alpha\left(c_{1} d\right)^{2} / 8\right)\|f\|_{1} \leqq \int T f(y)|y|^{2} \mu(d y) .
$$

Proof.

$$
\begin{aligned}
\int T f(y)|y|^{2} \mu(d y) & =\iint f(x) F(x, y)|y|^{2} \mu(d x) \mu(d y) \\
= & \iint f(x) F(x, y)|y-x|^{2} \mu(d x) \mu(d y)+\iint f(x) F(x, y) 2 x \cdot y \mu(d x) \mu(d y) \\
& -\iint f(x) F(x, y)|x|^{2} \mu(d x) \mu(d y) \\
= & \int f(x)\left[\int F(x, y)|y-x|^{2} \mu(d y)\right] \mu(d x)+\int T_{y}^{*}(f(x) 2 x \cdot y)(x) \mu(d x) \\
& -\int T_{x}\left(|x|^{2} f(x)\right)(y) \mu(d y) .
\end{aligned}
$$

Here $T_{y}^{*}$ means $T^{*}$ acting on functions of $y$, etc.

Of these three terms, the first is

$$
\begin{aligned}
& \geqq \int f(x)\left[\int_{S_{c_{1} d\left(x, R^{c}\right)(x)}} \alpha|y-x|^{2} m\left(c_{1} d\left(x, R^{c}\right)\right)^{-1} \mu(d y)\right] \mu(d x) \\
& \geqq \int_{E}\left(f(x) \alpha\left(c_{1} d\left(x, R^{c}\right)\right)^{2} / 8\right) \mu(d x) \geqq\left(\alpha\left(c_{1} d\right)^{2} / 8\right)\|f\|_{1},
\end{aligned}
$$

using (2.9). Since $f(x) 2 x \cdot y$ is harmonic in $y, T_{y}^{*}(f(x) 2 x \cdot y)(z)=f(x) 2 x \cdot z$, for all $z$. Thus the second of the three terms is $\int f(x) 2|x|^{2} \mu(d x)$. Since $\|T g\|_{1}=\|g\|_{1}$ for any $g \geqq 0$ in $\mathscr{L}_{1}(R, \mu)$, the third of the three terms is $\int f(x)|x|^{2} \mu(d x)$. Substituting, the lemma is proved.

Corollary (3.2). $\int T f(y)|y|^{2} \mu(d y) \geqq \int f(y)|y|^{2} \mu(d y)$, for every $f \geqq 0$ in $\mathscr{L}_{1}(R, \mu)$.

COROLlaRY (3.3). $\int T f(y)|y|^{2} \mu(d y) \geqq \int f(y)|y|^{2} \mu(d y)+\left(\alpha\left(c_{1} d\right)^{2} / 8\right)\left\|\chi_{E} f\right\|_{1}, \quad$ for every $f \geqq 0$ in $\mathscr{L}_{1}(R, \mu)$. 
COROllary (3.4).

$$
\begin{aligned}
\int T^{k} f(y)|y|^{2} \mu(d y) \geqq & \int f(y)|y|^{2} \mu(d y) \\
& +\left(\alpha\left(c_{1} d\right)^{2} / 8\right)\left[\left\|\chi_{E} f\right\|_{1}+\left\|\chi_{E} T f\right\|_{1}+\cdots+\left\|\chi_{E} T^{k-1} f\right\|_{1}\right],
\end{aligned}
$$

for every $f \geqq 0$ in $\mathscr{L}_{1}(R, \mu)$ and every $k \geqq 1$.

Since $\left|y^{2}\right|$ is bounded for $y \in R$, Corollary (3.4) shows that $T$ is dissipative. In particular,

(3.5) $\lim _{k \rightarrow \infty}\left\|\chi_{E} T^{k} f\right\|_{1}=0$ for every $f$ in $\mathscr{L}_{1}(R, \mu)$.

Lemma (3.6). Let $E \subset R$ be a measurable set such that $d\left(E^{c}, R^{c}\right)>0$. Then $\Gamma_{E}=\lim _{k \rightarrow \infty} T_{E}^{k}$ (strongly).

Proof. Follows at once from (3.5).

Corollary (3.7). If $f \geqq 0, f \in \mathscr{L}_{1}(R, \mu)$, then $\left\|\Gamma_{E} f\right\|_{1}=\|f\|_{1}$.

COROLlaRy (3.8). $\Gamma_{E}^{*}$ leaves unaltered any function left unaltered by $T^{*}$.

Lemma (3.9). Let $\lambda>0$ be fixed. Then a number $\gamma>0$ and an integer $k_{0}>0$ exist such that for any ball $S_{c}(p) \subset R$ with $d\left(S_{c}(p), R^{c}\right)=\lambda c$, and for any $f \geqq 0$ in $\mathscr{L}_{1}(R, \mu)$,

$$
\left(T \chi_{S_{c}(p)}\right)^{k_{0}} f \geqq \gamma m(c)^{-1}\left\|\chi_{S_{c}(p)} f\right\|_{1}
$$

on $S_{c}(p)$.

Proof. Fix $c>0$ and $p \in \mathbf{R}^{n}$. Define $U=U(c, p)$ on $\mathscr{L}_{1}\left(S_{c}(p), \mu\right)$ by

$$
U f(y)=\int_{S_{c}(p)} f(x) \alpha m\left(c_{1}(\lambda+2) c\right)^{-1} \chi_{S_{c_{1} \lambda c}(x)}(y) \mu(d x),
$$

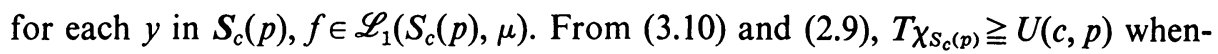
ever $S_{c}(p) \subset R$ and $d\left(S_{c}(p), R^{c}\right)=\lambda c$. We will establish the inequality for the operator $U(c, p)$. Clearly

$$
U f(y)=\alpha\left[c_{1}^{n}(\lambda+2)^{n} m(c)\right]^{-1} \int_{S_{c}(p) \cap S_{c_{1} \lambda c}(y)} f(x) \mu(d x) .
$$

Hence $U$ defines a continuous map from $\mathscr{L}_{1}\left(S_{c}(p), \mu\right)$ into $\mathscr{C}\left(S_{c}(p)\right)$, the Banach space of continuous functions on $\boldsymbol{S}_{c}(p)$.

Also, if $f$ is continuous on $S_{c}(p)$,

$$
\begin{aligned}
& \left\{y \mid y \in S_{c}(p), U f(y)>0\right\} \\
& \quad=\left\{y\left|y \in S_{c}(p),\right| y-x \mid<c_{1} \lambda c \text { for some } x \text { in } S_{c}(p) \text { with } f(x)>0\right\} .
\end{aligned}
$$

So by induction, for any $k \geqq 1$,

$$
\begin{aligned}
& \left\{y \mid y \in S_{c}(p), U^{k} f(y)>0\right\} \\
& \quad=\left\{y\left|y \in S_{c}(p),\right| y-x \mid<k c_{1} \lambda c \text { for some } x \text { in } S_{c}(p) \text { with } f(x)>0\right\} .
\end{aligned}
$$


Choose $k$ such that $k c_{1} \lambda>2$. Then for any continuous function $f$ on $S_{c}(p)$ which is nonnegative and nonzero at some point,

(3.14) $U^{k} f>0$ everywhere on $S_{c}(p)$.

Hence

(3.15) $U^{k+1} f>0$ everywhere on $S_{c}(p)$ for any $f \geqq 0$ in $\mathscr{L}_{1}\left(S_{c}(p), \mu\right), f \neq 0$.

For each $z \in S_{c}(p), y \in S_{c}(p)$, let

$$
G_{z}(y)=\alpha m\left(c_{1}(\lambda+2) c\right)^{-1} \chi_{S_{c_{1} \lambda c}(z)}(y) .
$$

Then $z \rightarrow G_{z}$ defines a continuous map from $S_{c}(p)$ into $\mathscr{L}_{1}\left(S_{c}(p), \mu\right)$. Hence $z \rightarrow U^{k+1} G_{z}$ defines a continuous map from $\boldsymbol{S}_{c}(p)$ into $\mathscr{C}\left(\boldsymbol{S}_{c}(p)\right)$. So, finally,

$$
z \rightarrow \inf _{y \in S_{c}(p)}\left[U^{k+1} G_{z}\right](y)
$$

defines a continuous map from $S_{c}(p)$ into $\mathbf{R}$. By (3.15) this last map is never zero. Hence there exists $\beta>0$ such that

(3.17) $U^{k+1} G_{z}>\beta$ on $S_{c}(p)$ for all $z \in S_{c}(p)$.

Let $f \geqq 0$ be in $\mathscr{L}_{1}\left(S_{c}(p), \mu\right)$. Let $h \geqq 0$ be in $\mathscr{L}_{\infty}\left(S_{c}(p), \mu\right)$.

$$
\begin{aligned}
& \int_{S_{c}(p)} h(y) U^{k+2} f(y) \mu(d y)=\int_{S_{c}(p)} U^{k+1 *} h(y) U f(y) \mu(d y) \\
& =\int_{S_{c}(p)} \int_{S_{c}(p)} U^{k+1 * h(y) f(z) G_{z}(y) \mu(d z) \mu(d y)}
\end{aligned}
$$

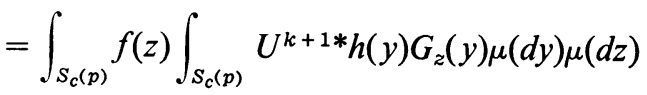

$$
\begin{aligned}
& \geqq \int_{S_{c}(p)} f(z) \mu(d z) \int_{S_{c}(p)} h(y) \beta \mu(d y) \\
& =\beta\|f\|_{1} \int_{S_{c}(p)} h(y) \mu(d y) \text {. }
\end{aligned}
$$

It follows from (3.18) that

(3.19) $U^{k+2} f \geqq \beta\|f\|_{1}$ on $S_{c}(p)$.

Let $k_{0}=k+2$. Let $\omega=\omega(c, p)$ be the largest number such that

(3.20) $U^{k_{0}} f \geqq \omega\|f\|_{1}$ on $S_{c}(p)$ for each $f \geqq 0$ in $\mathscr{L}_{1}\left(S_{c}(p), \mu\right)$. By (3.19), $\omega \geqq \beta>0$. We wish to show that $\omega(c, p) \mu\left(S_{c}(p)\right)$ is a constant, $\gamma$.

Obviously $\omega(c, p)$ is independent of $p, \omega(c, p)=\omega(c)$. For any $c>0, \sigma>0$, $f \in \mathscr{L}_{1}(R, \mu), f \geqq 0$,

$$
\begin{aligned}
& U(\sigma c, 0) f(y)=\alpha\left[c_{1}^{n}(\lambda+2)^{n} m(\sigma c)\right]^{-1} \int_{S_{\sigma c}(0) \cap S_{c_{1} \lambda \sigma c}(y)} f(x) \mu(d x) \\
& =\alpha\left[c_{1}^{n}(\lambda+2)^{n} m(c) \sigma^{n}\right]^{-1} \int_{S_{c}(0) \cap S_{c_{1} \lambda c}(y / \sigma)} f(\sigma z) \sigma^{n} \mu(d z)=U(c, 0) g(y / \sigma),
\end{aligned}
$$

where $g(x)=f(c x)$, and $g \in \mathscr{L}_{1}\left(S_{c}(0), \mu\right)$. 
Hence $U^{k}(\sigma c, 0) f(y)=U^{k}(c, 0) g(y / \sigma)$ for all $k \geqq 0$. Hence by (3.20),

(3.22) $U^{k_{0}}(\sigma c, 0) f \geqq \omega(c)\|g\|_{1}$ on $S_{\sigma c}(0)$, so $U^{k_{0}}(\sigma c, 0) f \geqq \omega(c) \sigma^{-n}\|f\|_{1}$. Hence $\omega(\sigma c) \geqq \omega(c) \sigma^{-n}$. Replacing $\sigma$ by $1 / \sigma$ and $c$ by $\sigma c, \omega(c) \geqq \omega(\sigma c) \sigma^{n}$. Hence $\omega(c)$ $=\omega(\sigma c) \sigma^{n}$ or $\omega(c) c^{n}$ is constant. Thus

(3.23) $\omega(c) \mu\left(S_{c}(p)\right)$ is constant, $=\gamma>0$.

Thus, rewriting (3.20), for any $c>0, p \in R, f \geqq 0$ in $\mathscr{L}_{1}\left(\mathbf{R}^{n}, \mu\right), \quad U^{k_{0}} f$ $\geqq \gamma m(c)^{-1}\left\|\chi_{S_{c}(p)} f\right\|_{1}$ on $S_{c}(p)$. Hence the lemma is proved.

Lemma (3.24). Let $\lambda>0$ be fixed. Then a number $\gamma>0$ exists such that for any ball $S_{c}(p) \subset R$ with $d\left(S_{c}(p), R^{c}\right) \geqq \lambda c$, and for any functions $f$ and $g$ in $\mathscr{L}_{1}(R, \mu)$ with $f=0=g$ outside $S_{c}(p)$, and $\int f d \mu=\int g d \mu ;$ if $r \leqq \lambda c$ then $\left\|\Gamma_{B^{r}}(f-g)\right\|_{1} \leqq(1-\gamma)\|f-g\|_{1}$.

Proof. We may assume $d\left(S_{c}(p), R^{c}\right)=\lambda c$, and also $f \geqq 0, g \geqq 0$, and $f g=0$.

$$
\left\|\Gamma_{B^{r}}(f-g)\right\|_{1}=\lim _{k \rightarrow \infty}\left\|\chi_{B^{r}} T_{B^{r}}^{k}(f-g)\right\|_{1} .
$$

Let $\gamma>0$ and $k_{0}$ be as in Lemma (3.9). Then

$$
\begin{aligned}
\left\|\Gamma_{B^{r}}(f-g)\right\|_{1} & \leqq\left\|T_{B^{r}}^{k_{0}}(f-g)\right\|_{1} \\
& \leqq\left\|\left[T_{B^{r}}^{k_{0}} f-\gamma m(c)^{-1}\|f\|_{1} \chi_{S_{c}(p)}\right]\right\|_{1}+\left\|\left[T_{B^{r}}^{k_{0}} g-\gamma m(c)^{-1}\|g\|_{1} \chi_{S_{c}(p)}\right]\right\|_{1},
\end{aligned}
$$

where each function in square brackets is nonnegative for $r \leqq \lambda c$. Hence

$$
\left\|\Gamma_{B^{r}}(f-g)\right\|_{1} \leqq\|f\|_{1}-\gamma\|f\|_{1}+\|g\|_{1}-\gamma\|g\|_{1}=\|f-g\|_{1}-\gamma\|f-g\|_{1} .
$$

Lemma (3.24) is the desired cancellation property of $T$.

4. Consequences of the smoothness of the boundary. It is assumed from now on that $\boldsymbol{R}$ is a compact $C^{1}$ manifold with boundary. More concretely,

(4.1) For each $x$ in $\partial R$, the boundary of $R$, there exists an open set $U$ containing $x$, and a $C^{1}$ function $f$ defined on $U$, such that $|\nabla f|^{2}>0$ on $U$, and $R \cap U$ $=\{y \mid y \in U, f(y)>0\}$.

LEMMA (4.2). For any $\varepsilon>0$, a number $\delta>0$ can be found such that, if $0<d<\delta$ and $x \in \partial R$, then a coordinate system $\left(y_{1}, \ldots, y_{n}\right)$ can be chosen, with $x$ as origin, such that $|y-x|<d, y_{n}>\varepsilon d$ imply $y \in R$, and $|y-x|<d, y_{n}<-\varepsilon d$ imply $y \in R^{c}$.

Lemma (4.2) is a direct consequence of assumption (4.1).

Lemma (4.3). Let $c_{2}>0$ and $\lambda>0$ be fixed. Then numbers $\delta>0$ and $c>0$ can be found such that, if $0<d<\delta$ and $x \in \partial R$, and $E=\left\{y\left|y \in B^{d}-B^{\lambda d},\right| y-x \mid<c_{2} d\right\}$, then $a$ ball $S$ of radius $c d$ can be found with $E \subset S \subset R$ and $d\left(S, R^{c}\right) \geqq(1 / 2) \lambda d$.

Lemma (4.3) follows from Lemma (4.2) and the compactness of $\boldsymbol{R}$.

Lemma (4.4). Let $\varepsilon>0$ be given. Then a number $c_{2}>0$ can be found such that, if $\theta$ is a function harmonic on $R$, continuous on $\boldsymbol{R}, 0 \leqq \theta \leqq 1$ on $\boldsymbol{R}$, and if for some $x$ in $R, \theta(y)=0$ whenever $y$ is in $\partial R$ and $|y-x|<c_{2} d\left(x, R^{c}\right)$, then $\theta(x)<\varepsilon$. 
Proof. Let $U=\left\{z \mid z_{1}^{2}+\cdots+z_{n-1}^{2}<1,0<z_{n}<1\right\}$. Let $\phi$ be a function harmonic on $U$, continuous and nonnegative on $\boldsymbol{U}$, such that

(4.5) $\phi=1$ on $\partial U \cap\left\{z \mid z_{n}>0\right\}$ and

(4.6) $\phi(0)=0$.

Then a number $\sigma>0$ exists such that $\phi(z)<\varepsilon$ for $|z|<\sigma$. Let

(4.7) $c_{2}=4 / \sigma+3$.

Choose $\delta>0$ such that, for any $d<\delta$, and any $x \in R$ with $d\left(x, R^{c}\right)=d$, a coordinate system $\left(y_{1}, \ldots, y_{n}\right)$ can be chosen with $x=\left(0, \ldots, 0, x_{n}\right),\left|x_{n}\right|<2 d$, and so that, for any $y$ with $|y|<5 d / \sigma$,

(4.8) $y_{n}>d$ implies $y$ is in $R$ and $y_{n}<d / 2$ implies $y$ is in $R^{c}$.

Suppose $\theta$ is a function harmonic on $R$, continuous on $\boldsymbol{R}$, and $0 \leqq \theta \leqq 1$ on $\boldsymbol{R}$; and, for some $x$ in $R, \theta(y)=0$ for all points $y$ in $\partial R$ with $|y-x|<c_{2} d, d<\delta$, where $d=d\left(x, R^{c}\right)$. It will be shown that $\theta(x)<\varepsilon$.

Using the coordinate system $y=\left(y_{1}, \ldots, y_{n}\right)$ just described, let

$$
U^{\prime}=\{y \mid y=(2 d / \sigma) z, z \in U\} \text {. }
$$

Clearly if $y \in U^{\prime}$, then

(4.9) $|y|<4 d / \sigma$ and $|y-x|<4 d / \sigma+2 d<c_{2} d$.

Hence $\theta(y)=0$ for all $y$ in $\partial R \cap \boldsymbol{U}^{\prime}$.

Let $\phi^{\prime}$ be defined on $U^{\prime}$ by $\phi^{\prime}(y)=\phi(\sigma y / 2 d) . \theta$ and $\phi^{\prime}$ are both defined on $\boldsymbol{U}^{\prime} \cap \boldsymbol{R}$. Consider $\partial\left(\boldsymbol{U}^{\prime} \cap \boldsymbol{R}\right)=\left(\partial U^{\prime} \cap \boldsymbol{R}\right) \cup\left(\boldsymbol{U}^{\prime} \cap \partial R\right)$. Clearly

$$
\partial U^{\prime} \cap \boldsymbol{R} \subset \partial U^{\prime} \cap\left\{y \mid y_{n} \geqq d / 2\right\} .
$$

Therefore $\phi^{\prime}=1$ on $\partial U^{\prime} \cap \boldsymbol{R}$. Also $\theta=0$ on $\boldsymbol{U}^{\prime} \cap \partial R$. Hence $\phi^{\prime} \geqq \theta$ on $\partial\left(\boldsymbol{U}^{\prime} \cap \boldsymbol{R}\right)$. Therefore $\phi^{\prime} \geqq \theta$ on $U^{\prime} \cap R$.

$$
\phi^{\prime}(x)=\phi\left(\left(0, \ldots, 0, \sigma x_{n} / 2 d\right)\right)
$$

and $\sigma x_{n} / 2 d<\sigma$. Therefore $\phi^{\prime}(x)<\varepsilon$ and, accordingly, $\theta(x)<\varepsilon$.

It was seen that the numbers $c_{2}$ and $\delta$ chosen are such that $c_{2}$ satisfies the requirements of the lemma, provided that we restrict our attention to $x$ in $R$ with $d\left(x, R^{c}\right)<\delta$. By choosing a new $c_{2}$, larger than the old, such that $c_{2} \delta$ is greater than the diameter of $R$, the proof is finished.

\section{A cancellation property for equivalent functions.}

(5.1) Two measures $\omega$ and $\nu$ on $R$ will be called equivalent if $\int h d \omega=\int h d \nu$ for every function $h$ which is harmonic on $R$, and continuous on $\boldsymbol{R}$. Two functions $f$ and $g$ in $\mathscr{L}_{1}(R, \mu)$ will be called equivalent if they are equivalent when regarded as (absolutely continuous) measures.

Lemma (5.2). For every measure $\boldsymbol{v}$ on $\boldsymbol{R}$, there is a unique measure $\hat{v}$ on $\partial R$ which is equivalent to $\nu$.

Proof. Since the Dirichlet problem is soluble for $R$ (because of (4.1)), Lemma (5.2) follows at once from the Riesz representation theorem. 
Lemma (5.3). Let $(X, \mathscr{F}, m)$ be a measure space, $m(X)<\infty$. Suppose for any set $A \in \mathscr{F}$ and any $\beta, 0 \leqq \beta \leqq 1$, that a set $B \in \mathscr{F}$ can be found such that $B \subset A$ and $m(B)$ $=\beta m(A)$. Now let $A_{1}, \ldots, A_{k}$ be fixed sets in $\mathscr{F}$. Let $E$ be the set of all points in $\boldsymbol{R}^{k}$ of the form $\left(m\left(P_{1}\right), \ldots, m\left(P_{k}\right)\right)$, where $P_{1}, \ldots, P_{k}$ are mutually disjoint sets in $\mathscr{F}$ with $P_{i} \subset A_{i}$ for $i=1, \ldots, k, \cup P_{i}=\bigcup A_{i}$. Then $E$ is compact.

Proof. The proof follows by induction on $k$, or by consideration of the partition generated by the sets $A_{1}, \ldots, A_{k}$.

Actually the same result holds for any finite measure space, but only the special case is needed here.

Lemma (5.4). A number $\gamma_{0}>0$ exists such that, if $f$ and $g$ are in $\mathscr{L}_{1}(R, \mu)$ and $f$ and $g$ are equivalent, then

$$
\lim _{r \rightarrow 0}\left\|\Gamma_{B^{r}}(f-g)\right\|_{1} \leqq\|f-g\|_{1}\left(1-\gamma_{0}\right) .
$$

\section{Proof.}

(5.5) By Lemma (4.4), a number $c_{2}>1$ can be chosen such that, if $\theta$ is a function harmonic on $R$, continuous on $\boldsymbol{R}, 0 \leqq \theta \leqq 1$ on $\boldsymbol{R}$, and if for some $x$ in $R, \theta(y)=0$ whenever $y$ is in $\partial R$ and $|y-x|<c_{2} d\left(x, R^{c}\right)$, then $\theta(x)<1 / 20$.

(5.6) By Lemma (2.24) a number $\lambda>0$ can be chosen such that, for any number $d>0$ and any $f \geqq 0$ in $\mathscr{L}_{1}(R, \mu)$,

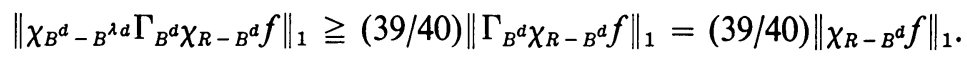

(5.7) By Lemma (4.3), numbers $\delta>0$ and $c>0$ can be chosen such that, if $0<d<\delta$, and $y \in \partial R$, and $E=\left\{z\left|z \in B^{d}-B^{\lambda d},\right| z-y \mid<2 c_{2} d\right\}$, then a ball $S$ of radius $c d$ can be found such that $E \subset S \subset R$ and $d\left(S, R^{c}\right) \geqq(1 / 2) \lambda d$.

(5.8) By Lemma (3.24) (with $\lambda$ replaced by $\lambda / 2 c$, a number $\gamma>0$ can be chosen such that, for any ball $S \subset R$ of radius $c d$ with $d\left(S, R^{c}\right) \geqq(1 / 2) \lambda d$, and for all functions $f$ and $g$ in $\mathscr{L}_{1}(R, \mu)$ with $f=0=g$ outside $S$ and $\int f d \mu=\int g d \mu$; if $r \leqq(1 / 2) \lambda d$, then $\left\|\Gamma_{B^{r}}(f-g)\right\|_{1} \leqq(1-\gamma)\|f-g\|_{1}$.

(5.9) Let $\gamma_{0}=(1 / 20) \gamma$.

Now let $f$ and $g$ be equivalent functions in $\mathscr{L}_{1}(R, \mu)$. To prove the lemma, it clearly can be assumed that $f \geqq 0, g \geqq 0$, and $f g=0$. Choose $d>0, d<\delta$ such that

$$
\left\|\chi_{B^{d}} f\right\|_{1} \leqq(1 / 39)\|f\|_{1}, \text { and }\left\|\chi_{B^{d}} g\right\|_{1} \leqq(1 / 39)\|g\|_{1} .
$$

It will be shown that for $r \leqq(1 / 2) \lambda d,\left\|\Gamma_{B^{r}}(f-g)\right\|_{1} \leqq\|f-g\|_{1}\left(1-\gamma_{0}\right)$, which will prove the lemma.

By (5.10) and (5.6),

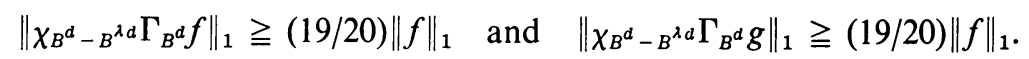

Let $\hat{v}$ be the measure on $\partial R$ which is equivalent to $f$ and $g$. Let $p_{1}, \ldots, p_{k}$ be points in $\partial R$ such that the collection of balls $S_{c_{2} d}\left(p_{i}\right), i=1, \ldots, k$, covers $\partial R$. Then the collection $S_{2 c_{2} d}\left(p_{i}\right), i=1, \ldots, k$, covers $B^{d}$, since $c_{2}>1$. 
Let $E_{i}, i=1, \ldots, k$, be an ordered partition of (all of) $\partial R$ such that

(5.12) $E_{i} \subset S_{c_{2} d}\left(p_{i}\right), i=1, \ldots, k$.

Let $\mathscr{C}$ be the class of all ordered partitions $\mathscr{P}=\left\{P_{1}, \ldots, P_{k}\right\}$ of (all of) $B^{d}$ such that

(5.13) $P_{i} \subset S_{2 c_{2} d}\left(p_{i}\right), i=1, \ldots, k$.

Because of the compactness property stated in Lemma (5.3), the sum

$$
\sum_{i=1}^{k}\left|\int_{P_{i}} \Gamma_{B^{d}} f d \mu-(1 / 2) \hat{\nu}\left(E_{i}\right)\right|^{2}
$$

defined for partitions $\mathscr{P} \in \mathscr{C}$ is minimal when $\mathscr{P}$ equals some partition $\mathscr{Q}$ $=\left\{Q_{1}, \ldots, Q_{k}\right\}$.

Let

$$
I^{+}=\left\{i \mid \int_{Q_{i}} \Gamma_{B^{d}} f d \mu>(1 / 2) \hat{v}\left(E_{i}\right)\right\} ; \quad I^{-}=\left\{i \mid \int_{Q} \Gamma_{B^{d}} f d \mu \leqq(1 / 2) \hat{v}\left(E_{i}\right)\right\}
$$

Because of the minimality of (5.14) when $\mathscr{P}=\mathscr{Q}$, it follows that, for any $i$ in $I^{+}$ and any $j$ in $I^{-}$,

$$
\int_{Q_{i} \cap S_{2 c_{2} d}\left(p_{j}\right)} \Gamma_{B^{d}} f d \mu=0
$$

Let $\theta$ be a function which is harmonic on $R$, continuous on $R, 0 \leqq \theta \leqq 1$ on $R$, such that

(5.17) $\theta=0$ on $\partial R$ outside $\bigcup_{i \in I^{-}} S_{c_{2} d}\left(p_{i}\right)$, and

(5.18) $\int \theta d \hat{v} \geqq \frac{3}{4} \sum_{i \in I^{-}} \hat{\nu}\left(E_{i}\right)$.

From (5.5),

(5.19) $\theta<(1 / 20)$ on $B^{d}$ outside $\bigcup_{i \in I^{-}} S_{2 c_{2} d}\left(p_{i}\right)$.

Hence from (5.16)

$$
\int_{Q_{\mathfrak{t}}} \theta \Gamma_{B^{d}} f d \mu \leqq(1 / 20) \int_{Q_{\mathfrak{t}}} \Gamma_{B^{d}} f d \mu
$$

for each $i$ in $I^{+}$.

Because $\Gamma_{B^{d}} f$ and $\hat{v}$ are equivalent,

$$
\int_{B^{d}} \theta \Gamma_{B^{d}} f d \mu \geqq \frac{3}{4} \sum_{i \in I^{-}} \hat{v}\left(E_{i}\right),
$$

by (5.18). But also

$$
\begin{aligned}
\int_{B^{d}} \theta \Gamma_{B^{d}} f d \mu & =\sum_{i=1}^{k} \int_{Q_{i}} \theta \Gamma_{B^{d}} f d \mu \\
& =\sum_{i \in I^{+}} \int_{Q_{i}} \theta \Gamma_{B^{d}} f d \mu+\sum_{i \in I^{-}} \int_{Q_{i}} \theta \Gamma_{B^{d}} f d \mu \leqq(1 / 20)\|f\|_{1}+\frac{1}{2} \sum_{i \in I^{-}} \hat{v}\left(E_{i}\right),
\end{aligned}
$$

using (5.20) and (5.15). 
Then from (5.21)

$$
\sum_{i \in I^{-}} \hat{v}\left(E_{i}\right) \leqq(1 / 5)\|f\|_{1} .
$$

Let $I^{*}$ denote the subset of $I^{+}$, consisting of those indices $i$ in $I^{+}$such that

$$
\int_{Q_{i} \cap\left(B^{d}-B^{\lambda d}\right)} \Gamma_{B^{d}} f d \mu \geqq(1 / 4) \hat{v}\left(E_{i}\right) .
$$

For $i \in I^{+}-I^{*}$, clearly

$$
\int_{Q_{i} \cap B^{\lambda d}} \Gamma_{B^{d}} f d \mu \geqq(1 / 4) \hat{v}\left(E_{i}\right)
$$

Therefore

$$
\frac{1}{4} \sum_{i \in I^{+}-I^{*}} \hat{v}\left(E_{i}\right) \leqq \int_{B^{\lambda d}} \Gamma_{B^{d}} f d \mu
$$

From (5.11) follows

(5.25) $\sum_{i \in I^{+}-I^{*}} \hat{v}\left(E_{i}\right) \leqq \frac{1}{5}\|f\|_{1}$.

From (5.22) and (5.25),

(5.26) $\sum_{i \in I^{*}} \hat{v}\left(E_{i}\right) \geqq \frac{3}{5}\|f\|_{1}$.

Repeating all these arguments for $g$ instead of $f$, a partition $\mathscr{Q}^{\prime}=\left\{Q_{1}^{\prime}, \ldots, Q_{k}^{\prime}\right\}$ of $B^{d}$ can be found such that

(5.27) $Q_{i}^{\prime} \subset S_{2 c_{2} d}\left(p_{i}\right), i=1, \ldots, k$, and a set of indices $I^{* \prime}$ can be found such that

(5.28) $\sum_{i \in I^{*}} \hat{\nu}\left(E_{i}\right) \geqq(3 / 5)\|g\|_{1}$ and

(5.29) $\int Q_{i}^{i n\left(B^{d}-B^{\lambda d}\right)} \Gamma_{B^{d}} g d \mu \geqq(1 / 4) \hat{v}\left(E_{i}\right)$ for each $i \in I^{* \prime}$.

Then

(5.30) $\sum_{i \in I^{*} \cap I^{*}} \hat{v}\left(E_{i}\right) \geqq(1 / 5)\|f\|_{1}=(1 / 5)\|g\|_{1}$.

For each $i \in I^{*} \cap I^{* \prime}$, let $S_{i}$ be a ball of radius $c d$ such that $S_{i} \subset R, S_{i}$ contains $S_{2 c_{2} d}\left(p_{i}\right) \cap B^{d}-B^{\lambda d}$, and $d\left(S_{i}, R^{c}\right) \geqq(1 / 2) \lambda d$. Such a ball exists by (5.7).

Then

(5.31) $Q_{i} \cap\left(B^{d}-B^{\lambda d}\right) \subset S_{i}$ and $Q_{i}^{\prime} \cap\left(B^{d}-B^{\lambda d}\right) \subset S_{i}$, for each $i$ in $I^{*} \cap I^{* \prime}$. Let

(5.32) $f_{i}=\chi_{Q_{i} \cap\left(B^{d}-B^{\lambda d}\right)} \Gamma_{B^{d}} f$ and $g_{i}=\chi_{Q_{i}^{i} \cap\left(B^{d}-B^{\lambda d}\right)} \Gamma_{B^{d}} g$, for each $i \in I^{*} \cap I^{* \prime}$.

From (5.23) and (5.29),

(5.33) $\left\|f_{i}\right\|_{1} \geqq(1 / 4) \hat{v}\left(E_{i}\right)$ and $\left\|g_{i}\right\|_{1} \geqq(1 / 4) \hat{v}\left(E_{i}\right)$.

Then by (5.8) and (5.33), for any $r \leqq(1 / 2) \lambda d$,

$$
\left\|\Gamma_{B^{r}}\left(f_{i}-g_{i}\right)\right\|_{1} \leqq\left\|f_{i}\right\|_{1}+\left\|g_{i}\right\|_{1}-(1 / 2) \gamma \hat{v}\left(E_{i}\right),
$$

for each $i$ in $I^{*} \cap I^{* \prime}$. Then

$$
\left\|\Gamma_{B^{r}} \Gamma_{B^{d}}(f-g)\right\|_{1} \leqq\left\|\Gamma_{B^{d}} f\right\|_{1}+\left\|\Gamma_{B^{d}} g\right\|_{1}-(1 / 2) \gamma \sum_{i \in I^{*} \cap I^{* *}} \hat{v}\left(E_{i}\right) .
$$

Hence

$$
\left\|\Gamma_{B^{r}}(f-g)\right\|_{1} \leqq\|f\|_{1}+\|g\|_{1}-(1 / 10) \gamma\|f\|_{1}
$$


by $(5.30)$ or

$$
\left\|\Gamma_{B^{r}}(f-g)\right\|_{1} \leqq\|f-g\|_{1}-(1 / 20) \gamma\|f-g\|_{1} .
$$

Since $\gamma_{0}=(1 / 20) \gamma$, the proof is finished.

COROLlaRY (5.38). If $f$ and $g$ are equivalent functions in $\mathscr{L}_{1}(R, \mu)$, then $\lim _{r \rightarrow 0}\left\|\Gamma_{B^{r}}(f-g)\right\|_{1}=0$.

(5.39) Proof of Theorem (2.11). For each $\varepsilon, 0<\varepsilon \leqq 1$, let

$$
G_{\varepsilon}(x, y)=m\left(\varepsilon d\left(x, R^{c}\right)\right)^{-1} \chi_{\left.S_{\varepsilon d(x, R} c\right)(x)}(y) .
$$

Clearly $G_{\varepsilon}(x, y)$ satisfies (2.1) and (2.3). Define $U_{\varepsilon}$ on $\mathscr{L}_{1}(R, \mu)$ by

$$
U_{\varepsilon} f(y)=\int f(x) G_{\varepsilon}(x, y) \mu(d x)
$$

for each $y \in R, f \in \mathscr{L}_{1}(R, \mu)$.

If $f$ is continuous and bounded, then clearly

(5.42) $\lim _{\varepsilon \rightarrow 0} U_{\varepsilon} f=f\left(\mathscr{L}_{1}\right.$-norm).

Since $\left\|U_{\varepsilon}\right\| \leqq 1$ for all $\varepsilon$, and $U_{\varepsilon}$ converges strongly to $I$ on a dense subset of $\mathscr{L}_{1}(R, \mu)$, it follows that

(5.43) $\lim _{\varepsilon \rightarrow 0} U_{\varepsilon} f=f$ for every $f \in \mathscr{L}_{1}(R, \mu)$.

Now let $h$ be in $\mathscr{L}_{\infty}(R, \mu)$ such that $T^{*} h=h$. Then

(5.44) $\Gamma_{B^{r}}^{*} h=h$ for any $r>0$,

so

(5.45) $\int h \Gamma_{B^{r}} f d \mu=\int h f d \mu$ for any $f$ in $\mathscr{L}_{1}(R, \mu)$, any $r>0$.

Let $\varepsilon_{1}$ and $\varepsilon_{2}$ be fixed, $0<\varepsilon_{1}, \varepsilon_{2} \leqq 1$. Clearly $G_{\varepsilon_{1}}(x, y)$ and $G_{\varepsilon_{2}}(x, y)$ are equivalent functions of $y$, for any fixed $x$. Then by Corollary (5.38),

(5.46) $\lim _{r \rightarrow 0}\left\|\Gamma_{B^{r}}\left(G_{\varepsilon_{1}}-G_{\varepsilon_{2}}\right)\right\|_{1}=0$.

Hence from (5.45),

(5.47) $\int h(y) G_{\varepsilon_{1}}(x, y) \mu(d y)=\int h(y) G_{\varepsilon_{2}}(x, y) \mu(d y)$ for every $x$.

From (5.41), (5.47) is just another way of writing

(5.48) $U_{\varepsilon_{1}}^{*} h=U_{\varepsilon_{2}}^{*} h$.

Letting $\varepsilon_{2} \rightarrow 0$ and using (5.43),

(5.49) $\int U_{\varepsilon_{1}}^{*} h f d \mu=\int h f d \mu$ for any $f$ in $\mathscr{L}_{1}(R, \mu)$.

\section{Hence}

(5.50) $U_{\varepsilon_{1}}^{*} h=h$.

Hence, from the definition of $U_{\varepsilon}^{*}, h$ is continuous and

(5.51) $\int h(y) G_{\varepsilon}(x, y) \mu(d y)=h(x)$ for any $x$ in $R$, or

$$
h(x)=m\left(\varepsilon d\left(x, R^{c}\right)\right)^{-1} \int h(y) \chi_{\left.S_{\varepsilon d(x, R} c\right)(x)}(y) \mu(d y) .
$$


Since $\varepsilon$ can have any value, $0<\varepsilon \leqq 1,(5.52)$ says $h$ has the (ordinary) mean value property at every point. Hence by a well-known result, $h$ is harmonic.

The author wishes to thank Professor M. A. Akcoglu for many helpful discussions concerning this problem.

\section{REFERENCE}

1. M. A. Akcoglu and R. W. Sharpe, Ergodic theory and boundaries, Trans. Amer. Math. Soc. 132 (1968), 447-460. MR 37 \#369.

Department of Mathematics, University of Minnesota, Minneapolis, Minnesota 55455 